Research article

\title{
Pinealocytes can not transport neurotropic viruses. Pinealo-to-retinal connection in prepubertal rats originates from pineal neurons: Light and electron microscopic immunohistochemical studies
}

\author{
Ágnes Csáki ${ }^{a}$, Katalin Köves ${ }^{\mathrm{a}, *}$, Anna L. Kiss ${ }^{\mathrm{a}}$, Pál Röhlich ${ }^{\mathrm{a}}$, Zsolt Boldogkői ${ }^{\mathrm{b}}$, \\ Viktória Vereczki ${ }^{\mathrm{a}}$, Zita Puskár ${ }^{\mathrm{a}}$, Dóra Tombácz ${ }^{\mathrm{b}}$, Zsolt Csabai ${ }^{\mathrm{b}}$ \\ ${ }^{a}$ Department of Anatomy, Histology and Embryology, Faculty of Medicine, Semmelweis University, Budapest, Hungary \\ ${ }^{\mathrm{b}}$ Department of Medical Biology, Faculty of Medicine, University of Szeged, Hungary
}

\section{A R T I C L E I N F O}

\section{Keywords:}

Rat

Transsynaptic tract tracing

Light and electron microscopy

Immunohistochemistry

\begin{abstract}
A B S T R A C T
It is well established that the adult mammalian pineal body (PB), with the exception of rodents, contains nerve cell bodies. Based on our previous results we have proposed that there is a pinealo-to-retinal neuronal connection in adult hamsters and in prebubertal rats. By the time the animals reached puberty, labeled cells in the PB were not observed in rats. In the present experiment, we provide light and electron microscopic immunohistochemical evidence that the labeled cells in the PB of prepubertal rats are neurons. Pinealocytes cannot transport neurotropic viruses. Virus labeled cells do not show S-antigen immunoreactivity typical for pinealocytes of six-day-old rats. Electron microscopic investigation confirmed the neuronal nature of virus labeled cells. These neurons, similarly to that of hamsters, also establish pinealo-to-retinal connections in prepubertal rats.
\end{abstract}

\section{Introduction}

It is well known that the pineal body (PB) of mammals evolutionarily derives from a double organ which is composed of the parietal eye and the PB [1]. The parietal eye, a wavelength discriminator, disappears during phylogenesis and only the $\mathrm{PB}$ is present in mammals. In this organ, the major cell types are pinealocytes and astrocytes. Pinealocytes produce melatonin and release it into the systemic circulation in a circadian rhythm [2]. The photosensitive image forming retina is in contact with the PB via a retinofugal neuronal chain. The last link is formed by sympathetic neurons located in the superior cervical ganglion (SCG) [3]. The presence of neurons in the PB is not generally accepted. The current opinion is that the $\mathrm{PB}$ in mammals is only an endocrine organ.

In contrast, neurons have been described in $\mathrm{PB}$ of various species of mammals such as humans [4], monkeys [5], cows [6], cats, hedgehogs, ferrets [7], and cotton rats [8]. Recently, we discovered nerve cell bodies in the PB of adult golden hamsters [9] and prepubertal rats [10]. A retrograde spreading virus, expressing green fluorescence protein (GFP), was injected into the vitreous body of the eye of adult hamsters and it labeled cells, besides other structures, in the PB. There were only a few labeled cells throughout the organ. In adult rats we did not find any labeling in the PB. However, in neonatal rats more than 300 virus labeled cells were counted. The number of virus labeled cells gradually decreased by the time of puberty [10]. After puberty no labeling was observed. Injection of an anterograde virus into PB of adult hamsters or six-day-old rats resulted in labeling in the retina $[9,10]$.

The aim of the present experiment was to provide evidence for the neuronal nature of the virus labeled cells in the prepubertal rat $\mathrm{PB}$ after eye inoculation using light (LM) and electron microscopic (EM) immunohistochemical methods. Because the pinealocytes also show some neuronal characteristics, we wanted to prove that pinealocytes were unable to transport neurotropic viruses.

\section{Materials and methods}

\subsection{Animals}

Wistar six-day-old and adult male rats were used for the experiment. The total number was 53. The animals were kept in an air-conditioned vivarium. They were fed with lab chow (Gödöllö, Hungary) and water. The rats were treated according to the rules of „EU Directive

\footnotetext{
* Corresponding author at: Department of Anatomy, Histology and Embryology, Semmelweis University, Túzoltó u. 58, 1094 Budapest, Hungary.

E-mail address: koves.katalin@med.semmelweis-univ.hu (K. Köves).
} 
2010/63/EU for animal experiments". Permission \#: 22.1/1158/3/ 2010.

\subsection{Neurotropic pseudorabies virus strains}

One of the viruses, expressing GFP (KA-GEI-memGFP-RV), could be transported only in retrograde direction. The other viruses expressing red fluorescence protein (Cherry or Tomato) were able to spread in both ante- and retrograde directions (VHS- $m C h e r r y-A-R V$ and KA-GEI-memTomato- $A-R V$ ). Specificity of virus strains were tested in our previous works $[9,10]$.

\subsection{Virus inoculation}

Six-day-old animals were anesthetized by ether. Adult animals were anesthetized by chloral hydrate (35 mg/100 g bw). Two $\mu \mathrm{L}$ of KA-GEImemGFP-RV $\left(8 \times 10^{8}\right.$ plaque forming unit $\left.(\mathrm{PFU}) / \mathrm{mL}\right)$ were injected at the corneo-scleral junction into the vitreous body of eye of six-day-old rats. The animals were sacrificed 2 days later. Adult rats received 0.5 $\mu \mathrm{L}$ of KA-GEI-memTomato- $A-R V$ into the PB or SCG. These animals were sacrificed 4 days later. The six-day-old animals received $0.5 \mu \mathrm{L}$ of VHS$m C h e r r y-A-R V$ into the $\mathrm{PB}$ and they were sacrificed 2 days later. The path, the virus had to take, is shorter in newborns than in adults. Intact animals did not receive virus.

\subsection{Stereotaxic intervention}

For injection of the virus into the $\mathrm{PB}$, adult animals were placed in a stereotaxic instrument. A glass capillary was fixed to an electrode holder. The tip of the glass capillary was lowered to the outer surface of the skull. The skull was then opened and the tip of glass capillary was lowered to the $\mathrm{PB}$ at the following parameters: $1 \mathrm{~mm}$ in front of the interaural line in the midline and $2 \mathrm{~mm}$ below the outer surface of the skull. In six-day-old animals, the virus was injected into the PB under visual control. All together 21 adult and 10 six-day-old animals were operated, but we have lost 18 adults and 7 newborns because the intervention was not in an appropriate place. Unfortunately, the confluens sinuum is just above the PB and the bleeding firmly disturbed the correct intervention.

\subsection{Experimental groups}

To prepare for LM studies, the animals were perfused with $4 \%$ paraformaldehyde (PFA) (Merck, Darmstadt, Germany) in potassium phosphate buffer (KPB) ( $\mathrm{pH} 7.4,0.1 \mathrm{~mol} / \mathrm{L})$. When processing for EM, the PFA contained $0.1 \%$ glutaraldehyde (GA) (Merck).

Group 1. Intact (no intervention or virus inoculation) six-day-old (No $=5$ ) and adult rats (No $=3$ ) were used for Nissl staining and LM immunohistochemistry.

Group 2. After KA-GEI-memGFP- $R V$ inoculation into the eye, six-dayold rats $(\mathrm{No}=5)$ were also used for LM immunohistochemistry.

Group 3. Six-day-old $(\mathrm{No}=3)$ and adult rats $(\mathrm{No}=3)$ received anteand retrograde spreading virus (VHS- $m C h e r r y-A-R V$ or KA-GEI-memTomato- $A-R V$ ) in the PB. Labeling was looked for in the retina.

Group 4. Adult rats $(\mathrm{No}=3)$ received Ka-GEI-memTomato- $A-R V$ in the SCG. Labeling was looked for in the PB.

Group. 5. Intact six-day-old rats $(\mathrm{No}=3)$ were perfused with PFA containing GA and were used for EM study.

Group 6. After virus inoculation with KA-GEI-memGFP- $R V$ in the eye, six-day-old rats (No $=3$ ) were perfused with PFA containing GA and were used for EM study.

\subsection{Preparing tissues for investigations}

After perfusion the brain, upper thoracic part of the spinal cord, SCG and eyes were removed, post-fixed overnight and washed in KPB. Then the organs were placed in sucrose (Merck) solution (15\%) for one day and embedded in Tissue-Tek (Sakura Finetek Europe B. V., Rijn, NL). Twenty- $\mu$ m-thick sections were cut with a Sandon cryotome. These sections were mounted on gelatinized slides for LM. The presence of virus was detected under a fluorescence microscope. Sections containing virus labeling were used for immunohistochemistry. Forty- $\mu$ m- thick sections were further processed and used for EM.

\subsection{Nissl-staining}

Some sections containing PB were stained with cresyl violet (Abcam, Cambridge, MA).

\subsection{LM immunohistochemistry}

Staining with only one antibody. Slides of the PB of animals, belonging to Group 1, were stained for glial fibrillary acidic protein (GFAP), S-antigen and neuron-specific nuclear protein (NeuN) immunoreactivities to make a distinction between astrocytes, pinealocytes and neurons. To enhance the penetration of antibodies, slides were treated with $1 \%$ Triton X-100 (Reanal, Hungary). Antibody to GFAP was produced in rabbit and purchased from Dako/Agilent (Santa Clara, CA) and applied in 1:100000 dilution. Immunoreactivities were visualized by ABC kit and diaminobenzidine tetrachloride (DAB) (Sigma-Aldrich, St. Louis, MO) reaction. S-antigen (arrestin-1) antibody was produced in rabbit and purchased from Abcam (Cambridge, MA) and applied in 1:1000 dilution. NeuN antibody was produced in mouse and purchased from Chemicon Int. (Temecula, CA) and applied in a dilution of 1:1000. Then the sections were incubated in anti-rabbit or anti-mouse fluorescence conjugate (Jackson Chemicals Ind, Gujarat, India) and applied in a dilution of 1:500 or ABC Kit and DAB reaction were used for visualization of the immunoreactive elements. The slides were studied and photographed with Olympus microscope.

Double labeling. Colocalization of S-antigen and KA-GEI-memGFP$R V$ or NeuN and KA-GEI-memGFP-RV labeling were investigated (Group 2). Fluorescence labeling was examined with multi-photon confocal microscopy (Radiance 2100 Rainbow Multiphoton Imaging System, BioRad Laboratories, coupled to an Eclipse E800 microscope, Nikon, USA) using the Zen 2012 software.

Positive and negative tests (by leaving out either the first, or, in other cases, the second antibody or both) were carried out.

\subsection{Pre-embedding for EM immunohistochemistry}

Forty- $\mu$ m-thick sections of the six-day-old virus inoculated rat $\mathrm{PB}$ (Group 6) were treated with borohydride (1\%, $10 \mathrm{~min}$ ), then with $\mathrm{H}_{2} \mathrm{O}_{2}$ $(3 \%, 20 \mathrm{~min})$. After washing and treatment with $10 \%$ goat serum, the slides were incubated with the primary antiserum against GFP in a dilution 1:2000. The antigen-antibody complex was visualized using $\mathrm{ABC}$ Kit and mild $\mathrm{DAB}$ reaction. The slides were processed for EM investigation.

\subsection{Embedding for EM study}

Slides of the PB of six-day-old intact and virus inoculated rats (Group 5 and 6) were dehydrated in ascending ethanol series then transferred to propylene oxide and embedded in Epon (Sigma-Aldrich). Semithin sections, stained with $1 \%$ toluidine blue (Reanal), were used for orientation then ultrathin sections were cut with a glass knife, mounted on grids, and allowed to dry. Uranyl acetate and lead citrate (Ted Pella Inc., Redding, CA) were used as post-embedding staining. The slides were studied by Jeol 1200 EX electron microscope. 

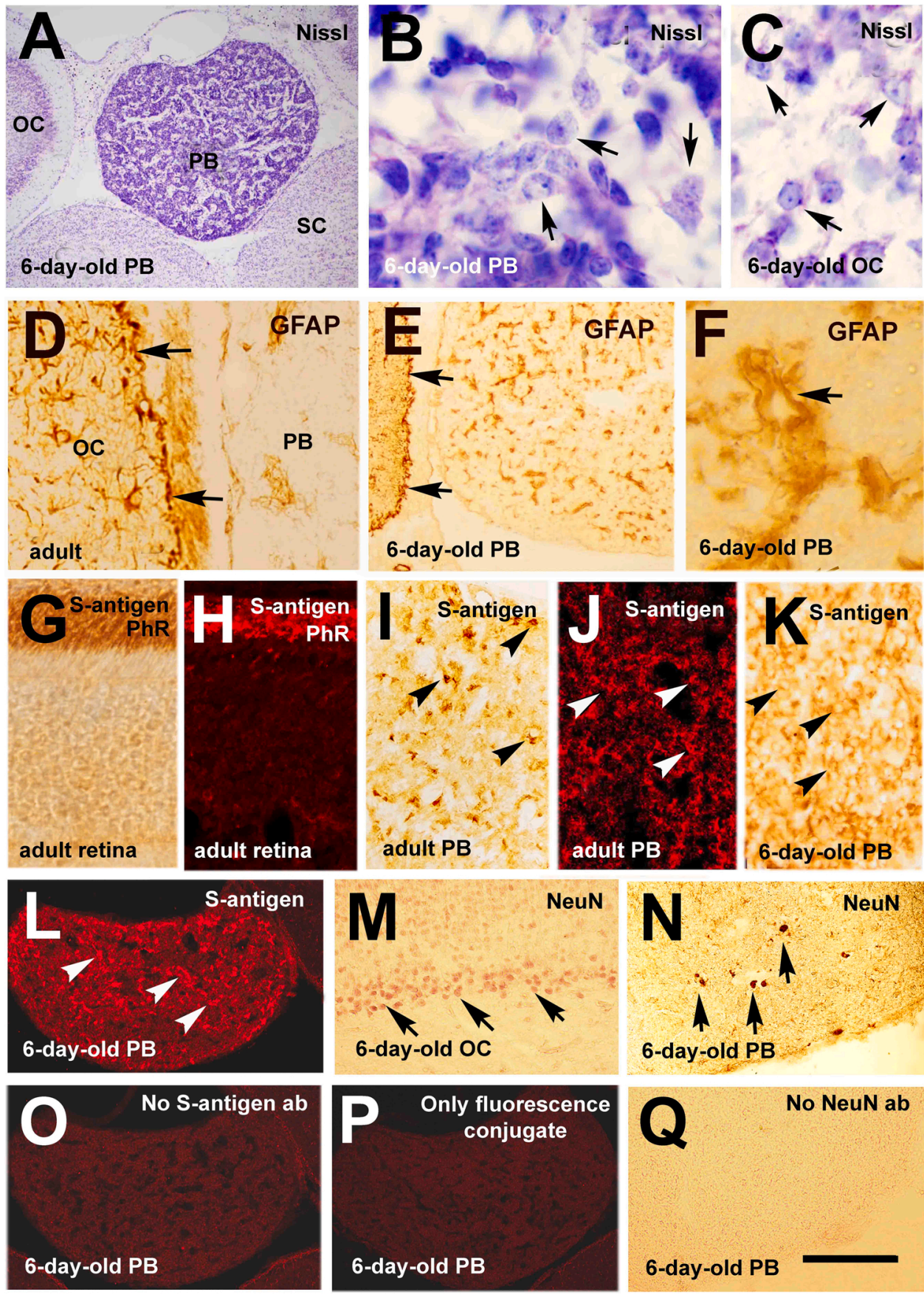
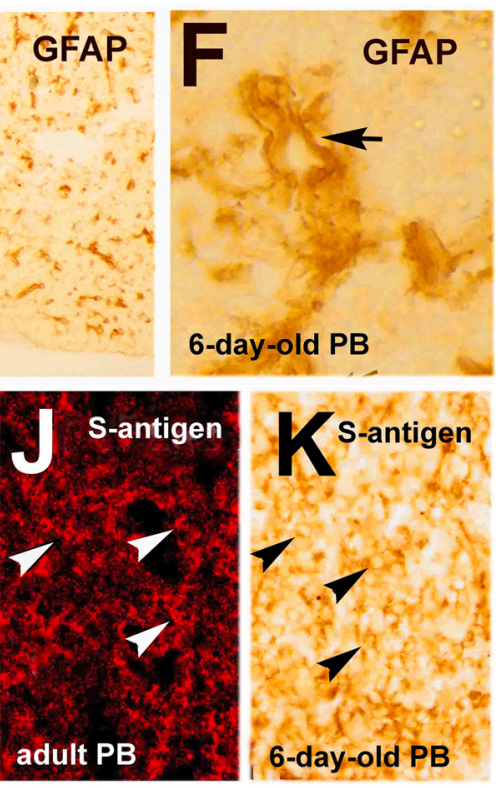

Fig. 1. Nissl-staining (A-C), GFAP (D-F), S-antigen (G-L) and NeuN immunostaining ( $M$ and $\mathrm{N})$ and specificity tests of S-antigen and NeuN immunostainings (O-Q). A. PB of a six-day-old rat in a frontal section. B. Pinealocytes and a group of neurons with very characteristic pale ball-like nucleus and prominent nucleolus. C. Nuclei of the neurons in the OC similar to those found in the PB. D-F. GFAP immunostained astrocytes in an adult and in a six-day-old rat PB. F. High power detail of E. G and H. S-antigen immunopositive PhRs in adult retina. I and J. Santigen immunopositive pinealocytes in adult PB. K and L. S-antigen immunopositive pinealocytes in six-day-old rat PB. M. NeuN immunopisitive neurons in the OC. N. NeuN immunopositive neurons in the $\mathrm{PB}$ of six-dayold rat. $\mathbf{O}$ and $\mathbf{P}$. In parallel sections leaving out the first antibody against S-antigen or the fluorescent conjugate prevented the immunostaining. Q. In a parallel section of $\mathrm{N}$ leaving out the antibodies prevented the NeuN immunostaining. Immunopositivity is visualized by $\mathrm{ABC}$ technique and DAB reaction (D-G, I, K, M, N and $\mathrm{Q})$ or by fluorescent staining $(\mathrm{H}, \mathrm{J}, \mathrm{L}, \mathrm{O}$ and P). Abbreviations: ab antibody, OC occipital cortex, PB pineal body, PhR photoreceptors, SC superior colliculus. Arrows point out nuclei of neurons (B, C, M and N), and superficial (D and E) and perivascular limiting membranes (F). Arrowheads indicate pinealocytes in I-L. Scale: $30 \mu \mathrm{m}$ in $\mathrm{F}, \mathrm{G}$ and $\mathrm{H} ; 40 \mu \mathrm{m}$ in B and $\mathrm{C} ; 200 \mu \mathrm{m}$ in $\mathrm{D}, \mathrm{I}-\mathrm{K}, \mathrm{M}, \mathrm{N}$ and Q; $300 \mu \mathrm{m}$ in $\mathrm{E} ; 600 \mu \mathrm{m}$ in $\mathrm{A}$, $\mathrm{L}, \mathrm{O}$ and $\mathrm{P}$.

\section{Results}

\subsection{LM investigations}

\subsubsection{Nissl staining (Group 1)}

The aim of this staining was to reveal cell nuclei typical for neurons. In the PB of adult rats most cells are pinealocytes. Nerve cells were not found. The PB of six-day-old rats is richly vascularized (Fig. 1A). The nucleus of pinealocytes is dark. The nuclei of nerve cells are pale with a centrally located prominent nucleolus and arranged in groups (Fig. 1B). The nucleus of these neurons is very similar to that of neurons located in the occipital cortex (Fig. 1C).

\subsubsection{Immunohistochemistry (Group 1)}

3.1.2.1. GFAP immunostaining. In adult rats the astrocytes showed GFAP immunoreactivity. The occipital cortex was used as positive control. Cell bodies of astrocytes were very rarely observed; however, their processes were evident. The astrocytic processes formed a well defined perivascular barrier. In the cortex, the superficial limiting membrane was also prominent (Fig. 1D). In six-day old rats, the perivascular membrane both in the cortex and the PB was also well defined and the perivascular membrane was even more prominent than in adults (Fig. 1E and F).

3.1.2.2. S-antigen immunostaining. Positive photoreceptor staining in the adult retina supports the specificity of our antibody (Fig. 1G and H). In adult rats a subpopulation of pinealocytes showed immunopositivity 

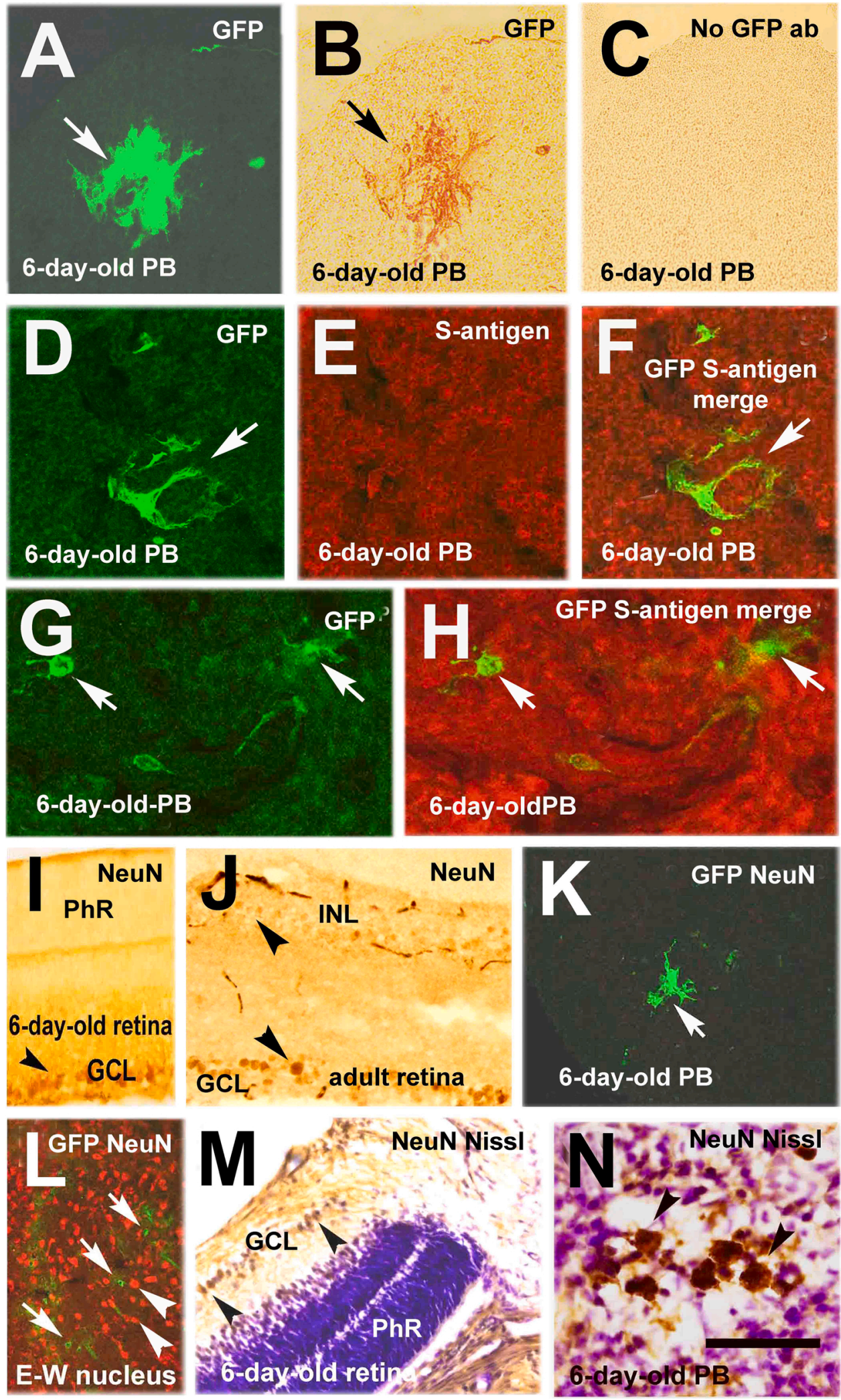

Fig. 2. KA-GEI-memGFP- $R V$ labeling from the eye in six-day-old rat PB and NeuN staining. A. GFP expressing virus labeled cells, identified under confocal microscope. B. Same virus labeled cells as in A stained with GFP antibody. C. In a parallel section, leaving out the first antibody, staining was not observed. $\mathbf{D}$ and $\mathbf{E}$. In the same sections virus labeling and S-antigen immunostaining. F. Merge image of the previous two. G and $\mathbf{H}$. Virus labeling and S-antigen immunostaining. There is no colocalization between virus labeling and S-antigen immunoreactivity. I. NeuN positive ganglion cells in the retina of a prepubertal rat. PhRs are negative. $\mathbf{J}$. NeuN staining in adult retina. Ganglion and amacrin cells show positive staining. $\mathbf{K}$ and $\mathbf{L}$. NeuN antibody (fluorescent staining) failed to stain GFP virus labeled cells. M and N. Cresyl violet failed to stain NeuN stained nuclei. Abbreviations: ab antibody, E-W Edinger-Westfal nucleus, GCL ganglion cell layer, INL inner nuclear layer, PB pineal body, PhR photoreceptor. Arrows show virus containing neurons, arrowheads show NeuN stained neurons. Scale: $60 \mu \mathrm{m}$ in $\mathrm{G}, \mathrm{H}$ and $\mathrm{N} ; 120 \mu \mathrm{m}$ in A-F, I and J; $240 \mu \mathrm{m} \mathrm{K}-$ M.
(Fig. 1I and J). Intensity of the staining varied considerably between individual cells. In six-day-old rats the pinealocytes were round and did not exhibit well definied processes (Fig. $1 \mathrm{~K}$ and L).

3.1.2.3. NeuN immunostaining. Positive staining in the occipital cortex (OC) confirms the specificity of our antibody (Fig. 1M). In PB of six-day- old rats, NeuN antibody also revealed neurons arranged in groups (Fig. 1N).

In the case of all the three antibodies the immunostaining was not successful, when we left out the first, in other cases the second biotinylated antibody, the $\mathrm{ABC}$ complex or the fluorescence conjugate in parallel sections. Fig.1O, P (S-antigen) and Q (NeuN) show the negative 
specificity tests. GFAP specificity test is not shown.

\subsubsection{KA-GEI-memGFP-RV inoculation of the eye of six-day-old rats} (Group 2)

Those sections were stained for GFP where virus labeling in the PB was previously identified under confocal microscope (Fig. 2A). The immunostaining was specific (Fig. 2B) because staining was not observed in adjacent section when we left out the primary (Fig. 2C), or secondary antibodies (not shown). Most labeled cells were arranged in groups. The neurons were well developed and presented long processes (Fig. 2D, G). Their appearance and size were very different from S-antigen positive pinealocytes (Fig. 2E, F, H). Co-localization was not found between S-antigen immunostaining and GFP virus labeling (Fig. 2D-F and G-H). Yellow colour, which would indicate the double labeling, was never observed. It means that GFP fluorescence did not appear in pinealocytes. Retinal photoreceptor cells were not stained by NeuN antibody in six-day-old rats; however, ganglionic cells were well stained (Fig. 1I and J). NeuN antibody was not able to stain virus labeled neurons (Fig. 2K), although the neurons were well stained in the adjacent OC (not shown). NeuN antibody was also unable to stain other virus inoculated neurons, even in the Edinger-Westfal nucleus of the midbrain because the nuclear protein was destroyed (Fig. 2L). When Nissl background staining was applied after NeuN staining, cresyl violet was not able to stain the nucleus of ganglion cells in the retina; however, other nuclei were very well stained (Fig. 2M). In the PB of six-day-old intact rats, cresyl violet was unable to stain neurons where NeuN immunostaining was carried out previously (Fig. 2N). There was a competition between the three nuclear stains.

3.1.4. VHS-mCherry-A-RV or KA-GEI-memTomato-A-RV injection into the $P B$ of six-day-old and adult rats (Group 3 and 4)

Animals in whom the injection site did not spread over the edge of the PB were included in this part of the study (Fig. 3A and D). In six-dayold rats, injection of VHS-mCherry- $A-R V$ into the $\mathrm{PB}$ always resulted in labeling in the retina (Fig. $3 \mathrm{~B}$ and $\mathrm{C}$ ). However, in adult rats KA-GEImemTomato-A-RV labeling was not found in the retina (Fig. $3 \mathrm{E}$ ) in spite
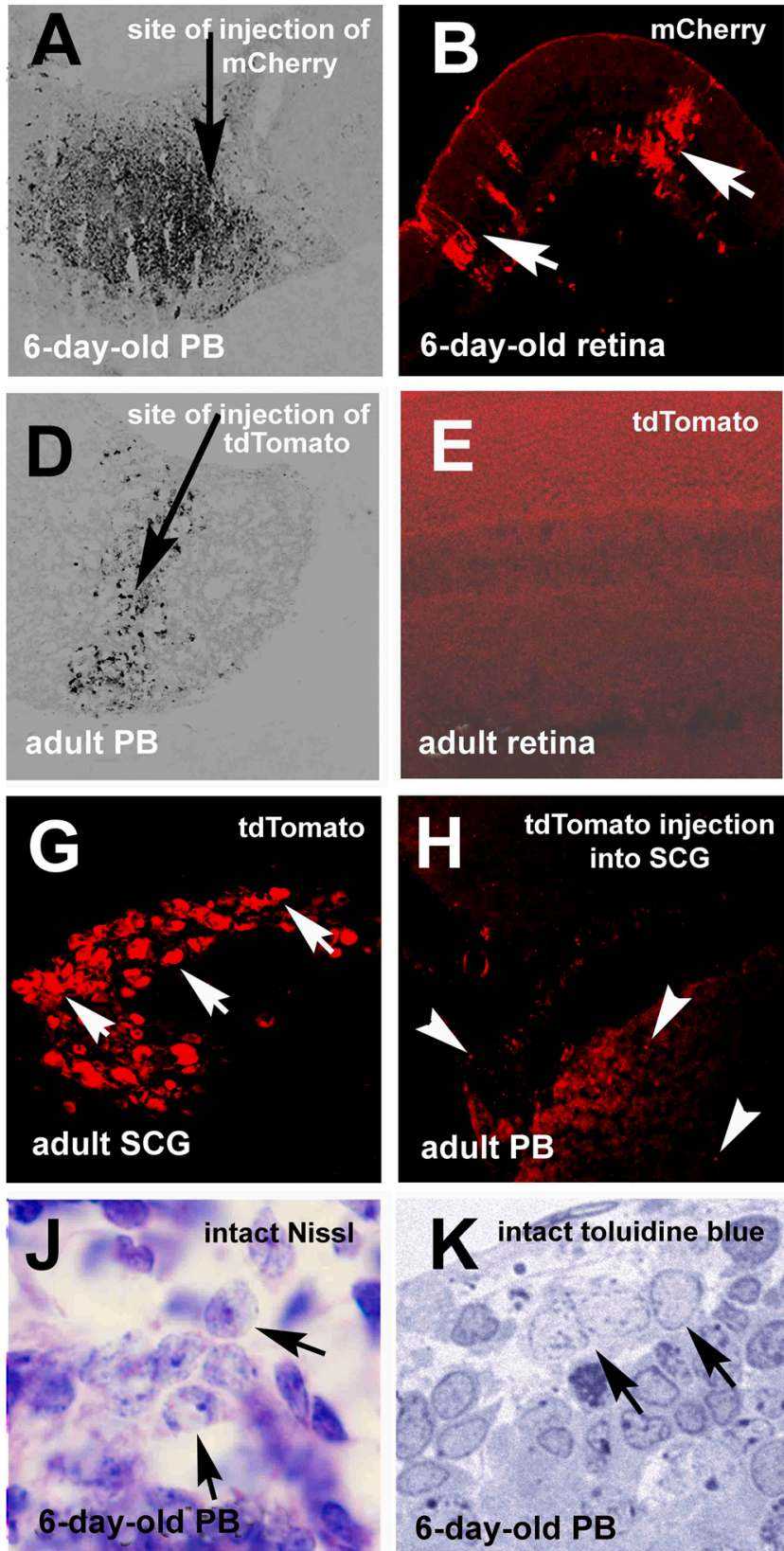
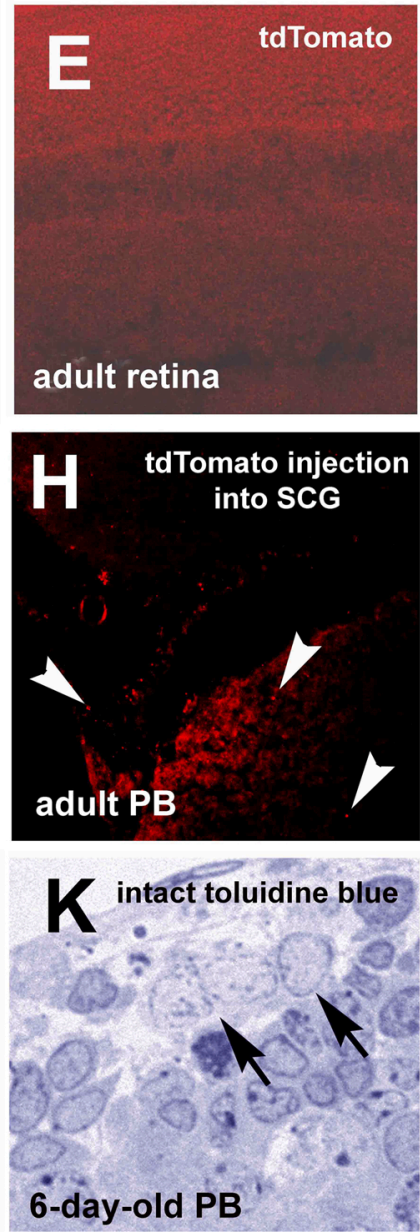
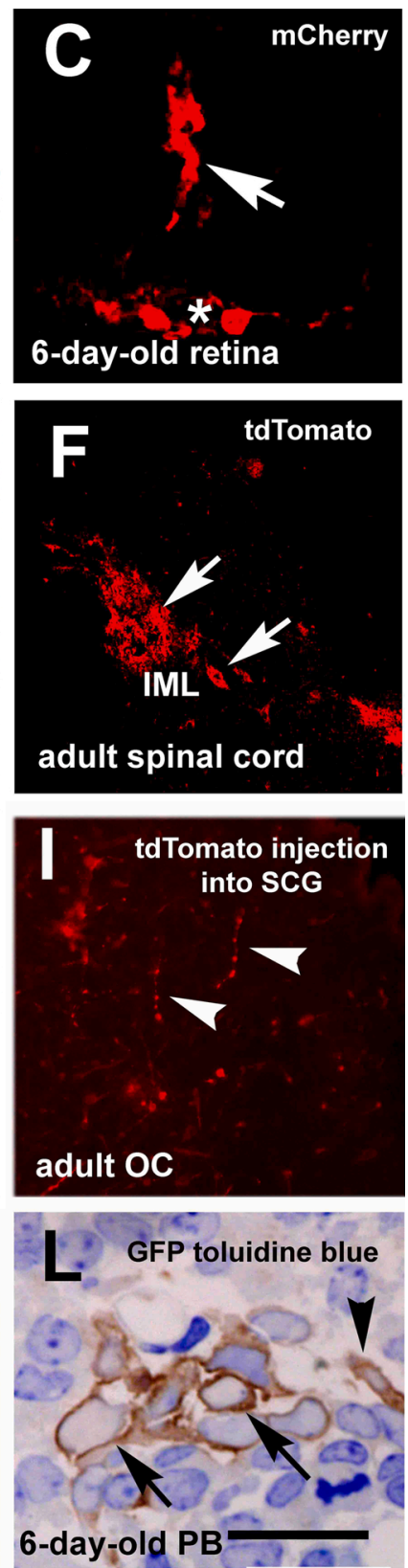

Fig. 3. Effect of ante-retrograde virus injection given into the PB and SCG (A-I). Neurons in the $\mathrm{PB}$ of six-day-old rats (J-L). A. The injection site in 6-day-old rat PB. B and C. Virus labeled cell columns in the retina of the same animal. $\mathbf{D}$. Injection site in adult rat PB. E. Virus labeling was not observed in the retina of the same animal. $\mathbf{F}$ and $\mathbf{G}$. Labeling in the IML and SCG of the same animal. $\mathbf{H}$. Fiber fragments in the pial surface and PB. Pinealocytes are not labeled. I. Fiber labeling in the adjacent OC. J. A group of neurons in the rostral pole of PB of an intact sixday-old rat. K. A group of neurons in a semithin section of PB of an intact rat. The nucleus of neurons is much paler than that of pinealocytes. L. Semithin section of a retrograde virus inoculated six-day-old rat $\mathrm{PB}$. Brown DAB reaction product indicates the presence of GFP expressing virus. Black and white arrows point out neurons. Arrowheads indicate fibers. Asterisk shows ganglion cells in the retina. Abbreviations: IML intermediolateral cell column, OC occipital cortex, PB pineal body, SCG superior cervical ganglion. Scale: $20 \mu \mathrm{m} \mathrm{K} ; 25 \mu \mathrm{m}$ in J and L; $75 \mu \mathrm{m}$ in E; $125 \mu \mathrm{m}$ in C and F-I; $250 \mu \mathrm{m}$ in $\mathrm{B} ; 300 \mu \mathrm{m}$ in $\mathrm{A}$ and $\mathrm{D}$. 


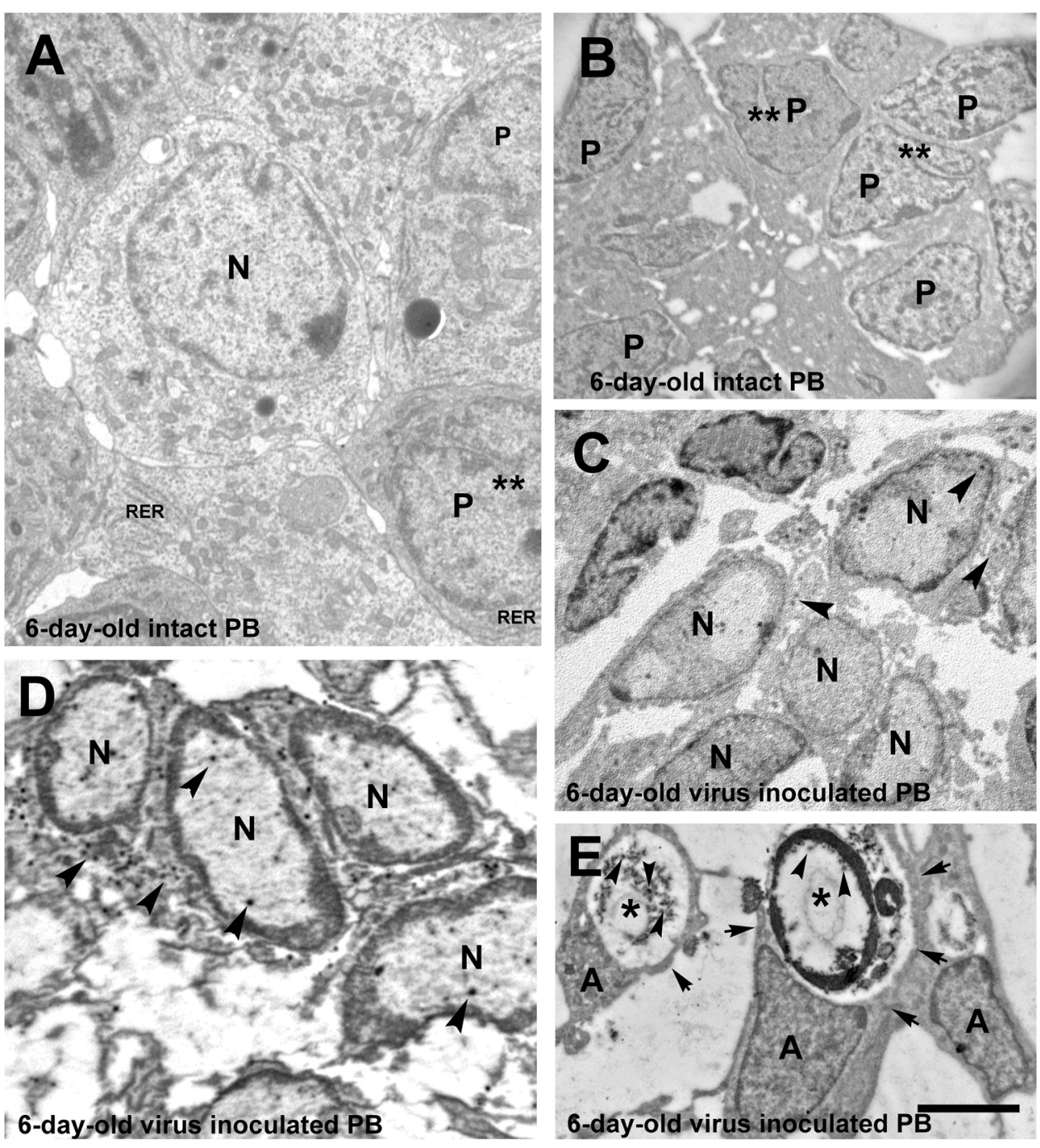

Fig. 4. EM images of six-day-old intact ( $\mathrm{A} n$ ad B) and KA-GEI-memGFP RV virus inoculated rat PBs (C-E). A. In the middle of the picture there is a neuron surrounded by pinealocytes. The nucleus of pinealocytes is characterized by deep infoldings of the nuclear membrane. B. A group of pinealocytes. C and D. Virus inoculated degenerated neurons. The nuclear membrane does not show infoldings. The cytoplasm is severely damaged and the cell boundaries are blurred. DAB granules are present in both nucleus and cytoplasm. E. Astrocytic processes ensheath $\mathrm{DAB}$ positive axons of virus inoculated neurons. Their nuclei are heterochromatic, DNA is tightly packed. Arrows point out astrocytic processes. Arrowheads show DAB particles which indicate the presence of viruses in $C, D$ and E. Single asterisks indicate $\mathrm{DAB}$ positive axons enveloped by astrocytic processes. Double asterisks show nuclear membrane infoldings of the pinealocytes. Abbreviations: A astrocyte, $\mathrm{N}$ neuron, $\mathrm{P}$ pinealocyte, $\mathrm{PB}$ pineal body, RER rough endoplasmic reticulum. Scale: $2 \mu \mathrm{m}$ in A; $4 \mu \mathrm{m}$ in. B-E. of the fact that the viability of the virus was confirmed by labeling in preand postganglionic sympathetic neurons (Fig. 3F and G). When the virus was injected into the SCG, labeled neurons were never observed in the $\mathrm{PB}$, although the whole organ was sectioned and investigated. Some labeled fiber fragments were only found in the PB (Fig. 3H), although well defined fiber staining was seen in the adjacent OC (Fig. 3I).

\subsection{EM investigations of six-day old intact and KA-GEI-memGFP-RV inoculated rats (Group 5 and 6)}

Similar to what was seen in Nissl stained slides (Fig. 3J), in semithin sections of untreated intact rat $\mathrm{PB}$, groups of neurons were observed (Fig. 3K). After inoculation of the eye, the presence of virus in the PB was visualized using GFP immunostaining. Brown DAB reaction product indicated the presence of the virus in semithin sections (Fig. 3L).

With electron microscopy, neurons of intact animals exhibit low electron density. Their round nuclei are euchromatic (Fig. 4A). The nuclei of pinealocytes are angular, usually triangular in shape and characterized by very deep infoldings of the nuclear membrane (Fig. 4A and B). After inoculation of the eye with KA-GEI-memGFP-RV, neurons in the PB became infected by the virus; however, pinealocytes remained negative. Moderately DAB positive neurons, organized in groups, were observed (Fig. 4C and D). Small dark uniform granules indicated the $\mathrm{DAB}$ positivity in both cytoplasm and nucleus. In infected neurons, the chromatin was attached to the inner surface of the nuclear membrane. The nuclei and the major part of the cytoplasm were damaged due to viral infection. The nuclei of astrocytes were ovoid or angular. In crosssections of infected axons, DAB positive granules were seen and the axons were encompassed by astrocytic processes (Fig. 4E).

\section{Discussion}

Data in the literature show that GFAP [11], S-antigen [12], and NeuN [13] immunostainings can be used as specific markers to distinguish between three cell types of the PB. To demonstrate the specificity of our antibodies, cortex and retina were also stained as reference tissues. GFAP positive astrocytes and NeuN positive neurons are characteristic cell types in the parietal cortex, and S-antigen immunoreactive photoreceptors are located in the retina. In our study, S-antigen positive pinealocytes and retinal photoreceptors did not show NeuN immunoreactivity, though ganglion cells were stained. The results support our view that these antibodies are suitable to distinguish between the three groups of cells.

S-antigen (a rhodopsin kinase) "downregulates" photo-excited rhodopsin [14]. S-antigen is present in the pinealocytes of adult [15] and newborn rats as well [16]. In adult PB, processes of pinealocytes may leave the $\mathrm{PB}$ and establish synaptic-like connection with nerve fibers in the habenula [12]. In our tissue samples, S-antigen antibody also stained pinealocytes in adult and six-day-old rats. Co-localization was not found between S-antigen immunoreactivity and viral labeling, indicating that pinealocytes were not able to take up and transport the virus. When a retrograde virus was injected into the eye of adult rats, 
labeling was not found anywhere in PB. In our previous material, we found labeled neurons in the habenula when a retrograde tracer was injected into the eye of adult hamsters and rats $[9,17]$. In spite of this fact, the virus could not pass through connections between the habenular neurons and the pinealocyte processes.

When the virus was transported in an anterograde manner from SCG, sympathetic nerve fibers were barely labeled in the $\mathrm{PB}$ and pinealocytes did not show labeling. These observations well correlate with the results published by Frank and co-workers [18]. They showed that autonomic fibers are well separated from pinealocytes by connective tissue septae and these fibers form vasomotor nerve endings. This observation further confirms our hypothesis that pinealocytes cannot take up and transport neurotropic viruses.

With the use of a virus, which can be transported in both ante- and retrograde manners, a pinealo-to-retinal pathway was identified in hamsters [9] and in prepubertal rats as well [10]. In this present study we also observed this pathway. When an anterograde virus was injected into six-day-old rat $\mathrm{PB}$, labeling was observed in the retina; however, after virus injection into adult rat $\mathrm{PB}$, retinal labeling was not found, even though the viability of the virus was confirmed by the presence of labeling in sympathetic pre- and postganglionic neurons.

EM investigations of the PB of intact and virus inoculated six-day-old rats strongly support our above-mentioned hypothesis that there are neurons in the PB of prepubertal rats. These neurons can be labeled by inoculation of eye with a retrograde spreading virus. In intact rats, the neurons are less electron dense than pinealocytes. In inoculated rats the plasma membrane of neurons was damaged and the cytoplasm was filled with electron lucent large vesicles. These cells were considered as neurons. Presence of DAB positive axons also clearly shows that these axons have to belong to neurons, cell bodies of which are located in the PB. This is explained by the fact that the virus, used for eye inoculation, could spread only in retrograde direction. The size of DAB particles in infected neuronal cells and axons $(200-250 \mathrm{~nm})$ well correlate with the size of pseudorabies virus [19].

\section{Conclusion}

We provided LM and EM evidence that the prepubertal rat PB contains neurons; however, the number of these neurons gradually decreases during prepubertal period. These neurons establish a pinealo-toretinal connection. Similar connection does not exist in adult rats because the adult PB does not contain neurons. Pinealocytes cannot take up and transport neurotropic viruses.

\section{Author statement}

Anna L. Kiss and Pál Röhlich were involved in the EM investigations, Zsolt Boldogkői, Dóra Tombácz and Zsolt Csabai were involved in the preaparation of the viruses, Viktória Vreczki and Zita Puskár were involved in testing the antobodies. Animal experiment, photography and writing the manuscript were carried out by Ágnes Csáki and Katalin Köves.

\section{Funding}

This research did not receive any specific grant from funding agencies in public, commercial.

\section{Acknowledgments}

We are very grateful to Anna Takáts, Zsuzsanna Vidra, Katalin Szemere and Dóczi Nikolet for their excellent technical assistance. This work was supported by the Department of Anatomy, Histology and Embryology, Semmelweis University, Budapest, Hungary and grant from the National Research, Development and Innovation Office, OTKA K128247 to ZB.

\section{References}

[1] A. Oksche, Survey of the development and comparative morphology of the pineal organ, Prog. Brain Res. 10 (1965) 3-29.

[2] R.J. Reiter, Pineal Melatonin: Cell Biology of Its Synthesis and of Its Physiological Interactions, Endocr. Rev. 12 (1991) 151-180.

[3] R. Teclemariam-Meshbach, G.J. Ter Horst, F. Postema, J. Wortel, R.M. Buijs, Anatomical demonstration of the suprachiasmatic Nucleus-Pineal Pathway, J. Comp. Neurol. 406 (1999) 171-182.

[4] R.Y. Moore, P. Sibony, Enkephalin-like immunoreactivity in neurons in the human pineal gland, Brain Res. 457 (1988) 395-398.

[5] T. Ichimura, T. Arikuni, P.H. Hashimoto, Fine-structural study of the pineal body of 3 monkeys (Macaca fuscata) with special reference to synaptic formations, Cell Tissue Res. 244 (1986) 569-576.

[6] C. Badiu, L. Badiu, M. Coculescu, H. Vilhardt, M. Møller, Presence of oxytocinergic neuronal-like cells in the bovine pineal gland: an immunocytochemical and in situ hybridization study, J. Pineal Res. 31 (2001) 273-280.

[7] I. Vigh-Teichmann, B. Vigh, Immunocytochemistry and calcium cytochemistry of the mammalian pineal organ: a comparison with retina and submammalian pineal organs, Microsc. Res. Tech. 21 (1992) 227-241. Review.

[8] S. Matsushima, Y. Sakai, Y. Hira, Y. Oomori, S. Daikoku, Immunohistochemical studies on sympathetic and non-sympathetic nerve fibers and neuronal cell bodies in the pineal gland of cotton rats, Sigmodon hispidus, Arch. Histol. Cytol. 57 (1994) 47-58.

[9] Á. Csáki, B. Vígh, V. Boldogkői, Á. Szél, K. Köves, Is a neuronal chain between the pineal body and the retina in rats and hamsters? Transneuronal tracing studies, Neurosci. Lett. 588 (2015) 1-6.

[10] Á. Csáki, V. Vereczki, Á. Lukáts, Z. Boldogkői, A. Sebestyén, Z. Puskár, K. Köves, Ontogenesis of the pinealo-retinal neuronal connection in albino rats, Neurosci. Lett. 665 (2018) 189-194.

[11] M. Schachner, S.K. Huang, P. Ziegelmüller, B. Bizzini, R. Taugner, Glial cells in the pineal gland of mice and rats. A combined immunofluorescence and electronmicroscopic study, Cell Tissue Res. 237 (1984) 245-252.

[12] H.-W. Korf, M. Moller, l. Gery, J.S. Zigler, D.C. Klein, Immunocytochemical demonstration of retinal S-antigen in the pineal organ of four mammalian species, Cell Tissue Res. 239 (1985) 81-85.

[13] V.V. Gusel'nikova, D.E. Korzhevskiy, NeuN as a neuronal nuclear antigen and neuron differentiation marker, Acta Naturae 7 (2015) 42-47.

[14] C. Pfister, M. Chabre, J. Plouet, V.V. Tuyen, Y. De Kozak, J.P. Faure, H. Kühn, Retinal S- antigen identified as the $48 \mathrm{~K}$ protein regulating light-dependent phosphodiesterase in rods, Science 228 (1985) 891-893.

[15] H.W. Korf, T. Sato, A. Oksche, Complex relationships between the pineal organ and the medial habenular nucleus-pretectal region of the mouse as revealed by Santigen immuno-cytochemistry, Cell Tissue Res. 261 (1990) 493-500.

[16] L.A. Donoso, C.F. Merryman, K.E. Edelberg, R. Naids, C. Kalsow, S-antigen in the developing retina and pineal gland: a monoclonal antibody study, Invest. Ophthalmol. Vis. Sci. 26 (1985) 561-567.

[17] V. Vereczki, K. Köves, A. Csáki, K. Grósz, G.E. Hoffman, G. Fiskum, Distribution of hypothalamic, hippocampal and other limbic peptidergic neuronal cell bodies giving rise to retinopetal fibers: anterograde and retrograde tracing and neuropeptide immunohistochemical studies, Neuroscience 140 (2006) 1089-1100.

[18] C.L. Frank, C. Dávid, S. Czirok, C. Vincze, M.J. Manzano, B. Vígh, Autonomic nerves terminating on smooth muscle cells of vessels in the pineal organ of various mammals, Acta Biol. Hung. 54 (2003) 233-240.

[19] L.E. Pomeranz, A.E. Reynolds, C.J. Hengartner, Molecular biology of pseudorabies virus: impact on neurovirology and veterinary medicine, Microbiol. Mol. Biol. Rev. 69 (2005) 462-500. 